\title{
POINCARÉ CHAOS FOR A HYPERBOLIC QUASILINEAR SYSTEM
}

\author{
M. AKHMET, M.O. FEN, M. TLEUBERGENOVA, AND A. ZHAMANSHIN
}

Received 22 January, 2019

\begin{abstract}
The existence of unpredictable motions in systems of quasilinear differential equations with hyperbolic linear part is rigorously proved. We make use of the topology of uniform convergence on compact sets and the contraction mapping principle to prove the existence of unpredictable motions. Appropriate examples with simulations that support the theoretical results are provided.
\end{abstract}

2010 Mathematics Subject Classification: 34D10; 34D20; 34H10

Keywords: hyperbolic quasilinear systems, Poincaré chaos, unpredictable solutions

\section{INTRODUCTION AND PRELIMINARIES}

A special type of Poisson stable trajectory called an unpredictable trajectory, which leads to Poincaré chaos in the quasi-minimal set, was introduced in the paper [1]. Moreover, the papers [2]-[4] were concerned with the unpredictable solutions of various types of quasilinear differential equations in which the matrix of coefficients admits eigenvalues all with negative linear part. In this study, we deal with the unpredictable solutions of quasilinear differential equations with hyperbolic linear part such that the matrix of coefficients admits eigenvalues both with negative and positive real parts.

Being based on the existence of only one special type of Poisson stable trajectory, the confirmation of the presence of Poincare chaos is easier compared to the other chaos types $[5,8,9]$ since their definitions require the interaction of infinitely many motions such as sensitivity and the existence of a dense set of infinitely many unstable periodic motions embedded in the chaotic attractor. Therefore, it can be accepted that Poincaré chaos is more advantageous from the applications point of view.

Throughout the paper the Euclidean norm for vectors and the norm induced by the Euclidean norm for square matrices will be utilized.

The definition of an unpredictable function is as follows [4].

Definition 1. A uniformly continuous and bounded function $\vartheta(t): \mathbb{R} \rightarrow \mathbb{R}^{p}$ is unpredictable if there exist positive numbers $\epsilon_{0}, \sigma$ and sequences $\left\{t_{n}\right\},\left\{u_{n}\right\}$ both of 
which diverge to infinity such that $\left\|\vartheta\left(t+t_{n}\right)-\vartheta(t)\right\| \rightarrow 0$ as $n \rightarrow \infty$ uniformly on compact subsets of $\mathbb{R}$ and $\left\|\vartheta\left(t+t_{n}\right)-\vartheta(t)\right\| \geq \epsilon_{0}$ for each $t \in\left[u_{n}-\sigma, u_{n}+\sigma\right]$ and $n \in \mathbb{N}$.

For the convenience of our discussions, we will call the convergence of the function's shift on compact subsets of $\mathbb{R}$ in Definition 1 as Poisson stability and the existence of the number $\epsilon_{0}$ as unpredictability property of the function. Thus, a function is unpredictable, if it is Poisson stable and admits the unpredictability property.

Compared to the paper [4], we simplified the proof for the unpredictability property in the present study, and this increases the novelty as well as the applicability of the results.

The main object of the present paper is the system of quasilinear differential equations

$$
x^{\prime}(t)=A x(t)+f(x(t))+g(t),
$$

where $x(t) \in \mathbb{R}^{p}, p$ is a fixed natural number, the function $f: \mathbb{R}^{p} \rightarrow \mathbb{R}^{p}$ is continuous in all of its arguments, $f(0,0, \ldots, 0)=(0,0, \ldots, 0), g: \mathbb{R} \rightarrow \mathbb{R}^{p}$ is a uniformly continuous and bounded function, and all eigenvalues of the constant matrix $A \in \mathbb{R}^{p \times p}$ have nonzero real parts. We assume that $\Re e\left(\lambda_{i}\right)<0, i=1,2, \ldots, q$, and $\Re e\left(\lambda_{i}\right)>0$, $i=q+1, \ldots, p$, where $1 \leq q<p, \lambda_{i}, i=1,2, \ldots, p$, are the eigenvalues of the matrix $A$, and $\Re e\left(\lambda_{i}\right)$ denotes the real part of the eigenvalue $\lambda_{i}$.

Our purpose is to prove that system (1.1) possesses a unique unpredictable solution, provided that the function $g(t)$ is unpredictable in accordance with Definition 1 and the solution is uniformly globally exponentially stable if all eigenvalues of the matrix $A$ have negative real parts. According to the results of paper [1], the existence of an unpredictable solution implies Poincaré chaos in the dynamics.

The following condition on system (1.1) is required.

(C1) There exists a positive number $L_{f}$ such that $\left\|f\left(x_{1}\right)-f\left(x_{2}\right)\right\|$

$$
\leq L_{f}\left\|x_{1}-x_{2}\right\| \text { for all } x_{1}, x_{2} \in \mathbb{R}^{p} \text {. }
$$

It is known that one can find a regular $p \times p$ matrix $B$ such that the transformation $x=B y$ reduces system (1.1) to the system

$$
y^{\prime}(t)=C y(t)+F(y)+G(t),
$$

where $C=B^{-1} A B, F(y)=B^{-1} f(B y)$, and $G(t)=B^{-1} g(t)$. In system (1.2), the matrix $C$ is of the form $\operatorname{diag}\left(C_{-}, C_{+}\right)$, where the eigenvalues of the $q \times q$ matrix $C_{-}$and $(p-q) \times(p-q) C_{+}$respectively have negative and positive real parts. Let us denote $F(y)=\left(F_{-}(y), F_{+}(y)\right)$ and $G(t)=\left(G_{-}(t), G_{+}(t)\right)$, where the vectorfunctions $F_{-}$and $G_{-}$are of dimension $q$ and the vector-functions $F_{+}$and $G_{+}$are of dimension $p-q$.

It can be verified that $F(0,0, \ldots, 0)=(0,0, \ldots, 0) \in \mathbb{R}^{p}$ and the function $F$ is Lipschitzian with the Lipschitz constant

$$
L_{F}=\|B\|\left\|B^{-1}\right\| L_{f},
$$


under the condition $(C 1)$, that is, $\left\|F\left(y_{1}\right)-F\left(y_{2}\right)\right\| \leq L_{F}\left\|y_{1}-y_{2}\right\|$ for all $y_{1}, y_{2} \in$ $\mathbb{R}^{p}$. Moreover, according to Corollary 5.1 [3], the function $G(t)$ is unpredictable if and only if $g(t)$ is unpredictable.

In the next section, the main result of the paper is formulated for system (1.2) and interpreted for system (1.1).

\section{MAIN RESULT}

One can confirm that there exist numbers $K \geq 1$ and $\alpha>0$ such that $\left\|e^{C_{-} t}\right\| \leq$ $K e^{-\alpha t}$ for all $t \geq 0$ and $\left\|e^{C_{+} t}\right\| \leq K e^{\alpha t}$ for all $t \leq 0$.

The following condition is required.

(C2) $\alpha-2 K L_{F}>0$.

According to the theory of differential equations [6,7], a function $\varphi(t)=\left(\varphi_{-}(t), \varphi_{+}(t)\right)$ which is bounded on the whole real axis is a solution of sytem (1.2) if and only if it satisfies the equations

$$
\begin{gathered}
\varphi_{-}(t)=\int_{-\infty}^{t} e^{C_{-}(t-s)}\left[F_{-}(\varphi(s))+G_{-}(s)\right] d s, \\
\varphi_{+}(t)=-\int_{t}^{\infty} e^{C_{+}(t-s)}\left[F_{+}(\varphi(s))+G_{+}(s)\right] d s .
\end{gathered}
$$

Let us denote

$$
H=\frac{2 K\left\|B^{-1}\right\| M_{g}}{\alpha-2 K L_{F}}
$$

where $M_{g}=\sup _{t \in \mathbb{R}}\|g(t)\|$.

The number $H$ defined by (2.2) will be used in the following lemma, which is concerned with the existence and uniqueness of Poisson stable solution of system (1.1).

Lemma 1. Suppose that the conditions $(C 1)$ and $(C 2)$ are valid. If the function $g(t)$ is Poisson stable, then system (1.1) possesses a unique Poisson stable solution $\omega(t)$ such that $\sup \|\omega(t)\| \leq\|B\| H$.

Proof. In the proof, we will show that system (1.2) possesses a unique Poisson stable solution by using the contraction mapping principle, and this implies the existence of a unique Poisson stable solution of system (1.1).

Because the function $g(t)$ is Poisson stable, there exists a sequence $\left\{t_{n}\right\}, t_{n} \rightarrow \infty$ as $n \rightarrow \infty$, such that $\left\|g\left(t+t_{n}\right)-g(t)\right\| \rightarrow 0$ as $n \rightarrow \infty$ uniformly on each compact subset of $\mathbb{R}$.

Let $U$ be the set of all uniformly continuous and uniformly bounded functions $\psi(t): \mathbb{R} \rightarrow \mathbb{R}^{p}$ such that $\|\psi\|_{0} \leq H$, where the norm $\|\cdot\|_{0}$ is defined by $\|\psi\|_{0}=$ 
$\sup \|\psi(t)\|$, and $\left\|\psi\left(t+t_{n}\right)-\psi(t)\right\| \rightarrow 0$ as $n \rightarrow \infty$ uniformly on each compact sub$t \in \mathbb{R}$ set of $\mathbb{R}$.

Define an operator $\Pi$ on $U$ through the equations

$$
\Pi_{-} \psi(t)=\int_{-\infty}^{t} e^{C_{-}(t-s)}\left[F_{-}(\psi(s))+G_{-}(s)\right] d s
$$

and

$$
\Pi_{+} \psi(t)=-\int_{t}^{\infty} e^{C_{+}(t-s)}\left[F_{+}(\psi(s))+G_{+}(s)\right] d s
$$

such that $\Pi \psi(t)=\left(\Pi_{-} \psi(t), \Pi_{+} \psi(t)\right)$.

First of all, we will show that $\Pi(U) \subseteq U$. Fix an arbitrary function $\psi(t)$ that belongs to $U$. For all $t \in \mathbb{R}$, we have

$$
\begin{gathered}
\left\|\Pi_{-} \psi(t)\right\| \leq \int_{-\infty}^{t}\left\|e^{C_{-}(t-s)}\right\|\left\|F_{-}(\psi(s))+G_{-}(s)\right\| d s \\
\leq \int_{-\infty}^{t} K\left(L_{F}\|\psi(s)\|+\left\|B^{-1}\right\| M_{g}\right) e^{-\alpha(t-s)} d s \leq \frac{1}{\alpha} K\left(L_{F} H+\left\|B^{-1}\right\| M_{g}\right)
\end{gathered}
$$

and

$$
\begin{gathered}
\left\|\Pi_{+} \psi(t)\right\| \leq \int_{t}^{\infty}\left\|e^{C_{+}(t-s)}\right\|\left\|F_{+}(\psi(s))+G_{+}(s)\right\| d s \\
\leq \int_{t}^{\infty} K\left(L_{F}\|\psi(s)\|+\left\|B^{-1}\right\| M_{g}\right) e^{\alpha(t-s)} d s \leq \frac{1}{\alpha} K\left(L_{F} H+\left\|B^{-1}\right\| M_{g}\right)
\end{gathered}
$$

Therefore, $\|\Pi \psi\|_{0} \leq \frac{2}{\alpha} K\left(L_{F} H+\left\|B^{-1}\right\| M_{g}\right)=H$.

Now, let us fix an arbitrary positive number $\epsilon$ and a compact subset $[a, b]$ of $\mathbb{R}$, where $b>a$. Suppose that $a_{0}$ and $b_{0}$ are numbers satisfying $a_{0}<a, b_{0}>b$ such that the inequalities

$$
\begin{gathered}
\frac{2}{\alpha} K\left(L_{F} H+\left\|B^{-1}\right\| M_{g}\right) e^{-\alpha\left(a-a_{0}\right)}<\frac{\epsilon}{4}, \\
\frac{2}{\alpha} K\left(L_{F} H+\left\|B^{-1}\right\| M_{g}\right) e^{\alpha\left(b-b_{0}\right)}<\frac{\epsilon}{4}
\end{gathered}
$$

are valid, and let $\gamma$ be a number such that

$$
\gamma>\frac{4 K\left(L_{F}+\left\|B^{-1}\right\|\right)}{\alpha} .
$$

There exists a natural number $n_{0}$ such that if $n \geq n_{0}$, then $\left\|g\left(t+t_{n}\right)-g(t)\right\|<\frac{\epsilon}{\gamma}$ and $\left\|\psi\left(t+t_{n}\right)-\psi(t)\right\|<\frac{\epsilon}{\gamma}$ for all $t \in\left[a_{0}, b_{0}\right]$.

Therefore, one can verify for $n \geq n_{0}$ and $t \in\left[a_{0}, b_{0}\right]$ that 


$$
\begin{aligned}
& \left\|\Pi_{-} \psi\left(t+t_{n}\right)-\Pi_{-} \psi(t)\right\| \\
& \leq \int_{-\infty}^{a_{0}}\left\|e^{C_{-}(t-s)}\right\|\left(\left\|F_{-}\left(\psi\left(s+t_{n}\right)\right)-F_{-}(\psi(s))\right\|+\left\|G_{-}\left(s+t_{n}\right)-G_{-}(s)\right\|\right) d s \\
& +\int_{a_{0}}^{t}\left\|e^{C_{-}(t-s)}\right\|\left(\left\|F_{-}\left(\psi\left(s+t_{n}\right)\right)-F_{-}(\psi(s))\right\|+\left\|G_{-}\left(s+t_{n}\right)-G_{-}(s)\right\|\right) d s \\
& \leq \frac{2}{\alpha} K\left(L_{F} H+\left\|B^{-1}\right\| M_{g}\right) e^{-\alpha\left(t-a_{0}\right)}+\frac{\epsilon}{\alpha \gamma} K\left(L_{F}+\left\|B^{-1}\right\|\right)\left(1-e^{-\alpha\left(t-a_{0}\right)}\right)
\end{aligned}
$$

and

$$
\begin{aligned}
& \left\|\Pi_{+} \psi\left(t+t_{n}\right)-\Pi_{+} \psi(t)\right\| \\
& \leq \int_{b_{0}}^{\infty}\left\|e^{C_{+}(t-s)}\right\|\left(\left\|F_{+}\left(\psi\left(s+t_{n}\right)\right)-F_{+}(\psi(s))\right\|+\left\|G_{+}\left(s+t_{n}\right)-G_{+}(s)\right\|\right) d s \\
& +\int_{t}^{b_{0}}\left\|e^{C_{+}(t-s)}\right\|\left(\left\|F_{+}\left(\psi\left(s+t_{n}\right)\right)-F_{+}(\psi(s))\right\|+\left\|G_{+}\left(s+t_{n}\right)-G_{+}(s)\right\|\right) d s \\
& \leq \frac{2}{\alpha} K\left(L_{F} H+\left\|B^{-1}\right\| M_{g}\right) e^{\alpha\left(t-b_{0}\right)}+\frac{\epsilon}{\alpha \gamma} K\left(L_{F}+\left\|B^{-1}\right\|\right)\left(1-e^{\alpha\left(t-b_{0}\right)}\right) .
\end{aligned}
$$

Now, using the inequalities (2.3) and (2.4), one can obtain for $t \in[a, b]$ that

$$
\left\|\Pi_{-} \psi\left(t+t_{n}\right)-\Pi_{-} \psi(t)\right\|<\frac{\epsilon}{2}
$$

and

$$
\left\|\Pi_{+} \psi\left(t+t_{n}\right)-\Pi_{+} \psi(t)\right\|<\frac{\epsilon}{2}
$$

provided that $n \geq n_{0}$. Hence, if $n \geq n_{0}$, then the inequality $\left\|\Pi \psi\left(t+t_{n}\right)-\Pi \psi(t)\right\|<$ $\epsilon$ is valid for all $t \in[a, b]$, and therefore, $\left\|\Pi \psi\left(t+t_{n}\right)-\Pi \psi(t)\right\| \rightarrow 0$ uniformly as $n \rightarrow \infty$ on each compact subset of $\mathbb{R}$.

Additionally, it can be shown that $\Pi \psi(t)$ is uniformly continuous since its derivative is bounded. Thus, $\Pi(U) \subseteq U$.

Next, we will show that the operator $\Pi: U \rightarrow U$ is contractive. Let $\psi_{1}(t)$ and $\psi_{2}(t)$ be functions in $U$. Then, we have that

$$
\begin{aligned}
& \left\|\Pi_{-} \psi_{1}(t)-\Pi_{-} \psi_{2}(t)\right\| \\
& \quad \leq \int_{-\infty}^{t}\left\|e^{C_{-}(t-s)}\right\|\left\|F_{-}\left(\psi_{1}(s)\right)-F_{-}\left(\psi_{2}(s)\right)\right\| d s \leq \frac{K L_{F}}{\alpha}\left\|\psi_{1}-\psi_{2}\right\|_{0}
\end{aligned}
$$

and

$$
\begin{aligned}
& \left\|\Pi_{+} \psi_{1}(t)-\Pi_{+} \psi_{2}(t)\right\| \\
& \quad \leq \int_{t}^{\infty}\left\|e^{C_{+}(t-s)}\right\|\left\|F_{+}\left(\psi_{1}(s)\right)-F_{+}\left(\psi_{2}(s)\right)\right\| d s \leq \frac{K L_{F}}{\alpha}\left\|\psi_{1}-\psi_{2}\right\|_{0} .
\end{aligned}
$$


Therefore, the inequality $\left\|\Pi \psi_{1}-\Pi \psi_{2}\right\|_{0} \leq \frac{2 K L_{F}}{\alpha}\left\|\psi_{1}-\psi_{2}\right\|_{0}$ holds, and it implies that the operator $\Pi: U \rightarrow U$ is contractive.

Thus, system (1.2) possesses a unique Poisson stable solution $\xi(t)$, and consequently, $\omega(t)=B \xi(t)$ is the unique Poisson stable solution of system (1.1). Moreover, we have $\sup \|\omega(t)\| \leq\|B\| H$, since $\sup \|\xi(t)\| \leq H$.

$t \in \mathbb{R}$

$$
t \in \mathbb{R}
$$

The next theorem is concerned with the unpredictable solution of system (1.1).

Theorem 1. Suppose that conditions $(C 1)$ and $(C 2)$ are valid. If the function $g(t)$ is unpredictable, then system (1.1) possesses a unique unpredictable solution. Moreover, the unpredictable solution is uniformly globally exponentially stable if all eigenvalues of the matrix A have negative real parts.

Proof. According to Lemma 1, system (1.1) possesses a unique Poisson stable solution $\omega(t)$. Therefore, to prove that system (1.1) possesses a unique unpredictable solution, it remains to show that $\omega(t)$ admits the unpredictability property.

Since $g(t)$ is an unpredictable function, there exist positive numbers $\epsilon_{0}, \sigma$ and sequences $\left\{t_{n}\right\},\left\{u_{n}\right\}$ both of which diverge to infinity such that $\left\|g\left(t+t_{n}\right)-g(t)\right\| \geq$ $\epsilon_{0}$ for each $t \in\left[u_{n}-\sigma, u_{n}+\sigma\right]$ and $n \in \mathbb{N}$.

Assume that $\inf _{n}\left\|\omega\left(u_{n}+t_{n}\right)-\omega\left(u_{n}\right)\right\|=0$. This contradicts to the equation

$$
\begin{gathered}
\omega\left(t+t_{n}\right)-\omega(t)=\omega\left(u_{n}+t_{n}\right)-\omega\left(u_{n}\right)+\int_{u_{n}}^{t} A\left(\omega\left(s+t_{n}\right)-\omega(s)\right) d s \\
+\int_{u_{n}}^{t}\left(f\left(\omega\left(s+t_{n}\right)\right)-f(\omega(s)) d s+\int_{u_{n}}^{t}\left(g\left(s+t_{n}\right)-g(s)\right) d s,\right.
\end{gathered}
$$

since at least one coordinate of $g\left(s+t_{n}\right)-g(s)$ is separated from 0 so that the norm of the last integral is separated from 0 , and the norms of the other terms tend to zero as $n \rightarrow \infty$ for each fixed $t$ from the interval $\left[u_{n}-\sigma, u_{n}+\sigma\right]$. Therefore, we have that

$$
\inf _{n}\left\|\omega\left(u_{n}+t_{n}\right)-\omega\left(u_{n}\right)\right\|=2 \bar{\epsilon}_{0}
$$

for some positive number $\bar{\epsilon}_{0}$.

Now, fix a positive number $\eta$ such that

$$
\eta\left(\|A\|\|B\| H+\|B\| H L_{f}+M_{g}\right) \leq \frac{\bar{\epsilon}_{0}}{2},
$$

where $H$ is the number defined by (2.2). Using the inequality

$$
\begin{gathered}
\left\|\omega\left(t+t_{n}\right)-\omega(t)\right\| \geq\left\|\omega\left(u_{n}+t_{n}\right)-\omega\left(u_{n}\right)\right\|-\left|\int_{u_{n}}^{t}\|A\|\left\|\omega\left(s+t_{n}\right)-\omega(s)\right\| d s\right| \\
-\mid \int_{u_{n}}^{t} \| f\left(\omega\left(s+t_{n}\right)\right)-f\left(\omega(s)\left\|d s|-| \int_{u_{n}}^{t}\right\| g\left(s+t_{n}\right)-g(s) \| d s \mid,\right.
\end{gathered}
$$


one can obtain for $t \in\left[u_{n}-\eta, u_{n}+\eta\right]$ that

$$
\left\|\omega\left(t+t_{n}\right)-\omega(t)\right\| \geq 2 \bar{\epsilon}_{0}-2 \eta\left(\|A\|\|B\| H+\|B\| H L_{f}+M_{g}\right) \geq \bar{\epsilon}_{0} .
$$

Hence, the solution $\omega(t)$ is unpredictable.

On the other hand, one can show in a similar way to the proof of Theorem 4.1 [2] that if all eigenvalues of the matrix $A$ have negative real parts, then the unpredictable solution of system (1.1) is uniformly globally exponentially stable under the condition $(C 2)$.

The following two lemmas, which will be utilized in the Section 3, increases the applicability of our results.

Lemma 2. Suppose that $\phi(t): \mathbb{R} \rightarrow \mathbb{R}^{p}$ is an unpredictable function and $\zeta(t)$ : $\mathbb{R} \rightarrow \mathbb{R}^{p}$ is a continuous and periodic function. Then the function $\phi(t)+\zeta(t)$ is unpredictable.

Proof. Because the function $\phi(t)$ is unpredictable there exist positive numbers $\epsilon_{0}$, $\sigma$ and sequences $\left\{t_{n}\right\},\left\{u_{n}\right\}$ both of which diverge to infinity such that $\| \phi\left(t+t_{n}\right)-$ $\phi(t) \| \rightarrow 0$ as $n \rightarrow \infty$ uniformly on compact subsets of $\mathbb{R}$ and $\left\|\phi\left(t+t_{n}\right)-\phi(t)\right\| \geq \epsilon_{0}$ for each $t \in\left[u_{n}-\sigma, u_{n}+\sigma\right]$ and $n \in \mathbb{N}$. Let us denote $h(t)=\phi(t)+\zeta(t)$.

Due to the periodicity of $\zeta(t)$, there exists a subsequence of $\left\{t_{n}\right\}$ (we will assume without loss of generality that it is the sequence $\left\{t_{n}\right\}$ itself) such that $\left\|\zeta\left(t+t_{n}\right)-\zeta(t)\right\|$ $\rightarrow 0$ uniformly on the real axis. Hence, $\left\|h\left(t+t_{n}\right)-h(t)\right\| \rightarrow 0$ uniformly as $n \rightarrow \infty$ on compact subsets of $\mathbb{R}$. Again, because of the periodicity, one can find a subsequence of $\left\{u_{n}\right\}$, let us say the sequence itself, such that $\left\|\zeta\left(t+t_{n}\right)-\zeta(t)\right\|<\epsilon_{0} / 2$ if $t \in\left[u_{n}-\sigma, u_{n}+\sigma\right]$. Thus, $\left\|h\left(t+t_{n}\right)-h(t)\right\| \geq\left\|\phi\left(t+t_{n}\right)-\phi(t)\right\|-\| \zeta\left(t+t_{n}\right)-$ $\zeta(t) \|>\epsilon_{0}-\epsilon_{0} / 2=\epsilon_{0} / 2$ for $t \in\left[u_{n}-\sigma, u_{n}+\sigma\right]$.

Lemma 3. Suppose that $\phi(t): \mathbb{R} \rightarrow \mathbb{R}$ is an unpredictable function. Then the function $\phi^{3}(t)$ is unpredictable.

Proof. One can find numbers $\epsilon_{0}>0, \sigma>0$ and sequences $\left\{t_{n}\right\},\left\{u_{n}\right\}$ both of which diverge to infinity such that $\left\|\phi\left(t+t_{n}\right)-\phi(t)\right\| \rightarrow 0$ as $n \rightarrow \infty$ uniformly on compact subsets of $\mathbb{R}$ and $\left\|\phi\left(t+t_{n}\right)-\phi(t)\right\| \geq \epsilon_{0}$ for each $t \in\left[u_{n}-\sigma, u_{n}+\sigma\right]$ and $n \in \mathbb{N}$. The proof for the Poisson stability of $\phi^{3}(t)$ is trivial, since it follows from the uniform continuity of the cubic function on a compact set.

Fix a natural number $n$. To prove the unpredictability property, it is sufficient to show for $t \in\left[u_{n}-\sigma, u_{n}+\sigma\right]$ that $\left\|\phi\left(t+t_{n}\right)-\phi(t)\right\| \geq \epsilon_{0}$ implies $\| \phi^{3}\left(t+t_{n}\right)-$ $\phi^{3}(t) \| \geq \epsilon\left(\epsilon_{0}\right)$ for some positive number $\epsilon\left(\epsilon_{0}\right)$. We have that

$$
\begin{gathered}
\left|\phi^{3}\left(t+t_{n}\right)-\phi^{3}(t)\right|=\frac{1}{2}\left|\phi\left(t+t_{n}\right)-\phi(t)\right|\left[\phi^{2}\left(t+t_{n}\right)+\phi^{2}(t)\right. \\
\left.+\left(\phi\left(t+t_{n}\right)+\phi(t)\right)^{2}\right] \geq\left(\phi^{2}\left(t+t_{n}\right)+\phi^{2}(t)\right) \frac{\epsilon_{0}}{2} .
\end{gathered}
$$


Consider the function $F(a, b)=a^{2}+b^{2}$ for $|a-b| \geq \epsilon_{0}$. The minimum of $F$ occurs at the points $(a, b)$ with $|a|=|b|=\epsilon_{0} / 2$. Therefore, $\left|\phi^{3}\left(t+t_{n}\right)-\phi^{3}(t)\right| \geq \epsilon_{0}^{3} / 4$ for $t \in\left[u_{n}-\sigma, u_{n}+\sigma\right]$.

\section{EXAMPLES}

One of the possible ways to confirm the presence of chaos is through simulations. The concept of unpredictable solutions maintain the series of oscillations, but from the other side the chaos accompanies unpredictability. Consequently, we can look for a confirmation of the results for unpredictability observing irregularity in simulations. The approach is effective for asymptotically stable unpredictable solutions, and it is just illustrative for hyperbolic systems with unstable solutions. In the latter case we rely on the fact that any solution becomes unpredictable ultimately.

In the following examples we will utilize the function $\Theta(t): \mathbb{R} \rightarrow \mathbb{R}$ defined by

$$
\Theta(t)=\int_{-\infty}^{t} e^{-2(t-s)} \Omega(s) d s,
$$

which was discussed in paper [4]. In (3.1), the function $\Omega(t)$ is defined by $\Omega(t)=\psi_{i}$ for $t \in[i, i+1), i \in \mathbb{Z}$, where $\left\{\psi_{i}\right\}, i \in \mathbb{Z}$, is an unpredictable solution of the logistic map

$$
\lambda_{i+1}=3.91 \lambda_{i}\left(1-\lambda_{i}\right)
$$

inside the unit interval $[0,1]$. The function $\Theta(t)$ is bounded on the whole real axis such that $\sup _{t \in \mathbb{R}}|\Theta(t)| \leq 1 / 2$, and it is uniformly continuous since its derivative is bounded. It was proven in paper [4] that $\Theta(t)$ is an unpredictable function.

Example 1. Consider the system

$$
\begin{aligned}
& x_{1}^{\prime}=-3 x_{1}+2 x_{2}-0.01 x_{2}^{3}+8 \Theta(t)-0.2 \sin (3 t) \\
& x_{2}^{\prime}=x_{1}-2 x_{2}+0.05 x_{1}^{2}-5 \Theta(t)+3 \cos (3 t)
\end{aligned}
$$

where $\Theta(t)$ is the unpredictable function defined by (3.1). The eigenvalues of the matrix of coefficients of system (3.3) are -1 and -3 . One can confirm by means of Lemma 2 that the perturbation function $(8 \Theta(t)-0.2 \sin (3 t),-5 \Theta(t)+3 \cos (3 t))$ is unpredictable. According to Theorem 1 , there is a unique asymptotically stable unpredictable solution $\left(\varphi_{1}(t), \varphi_{2}(t)\right)$ of system (3.3). Consequently, any solution of the equation behaves irregularly ultimately. This is seen from the simulation of the solution with $x_{1}(0)=1,18, x_{2}(0)=1,01$ in Figures 1 and 2 . 

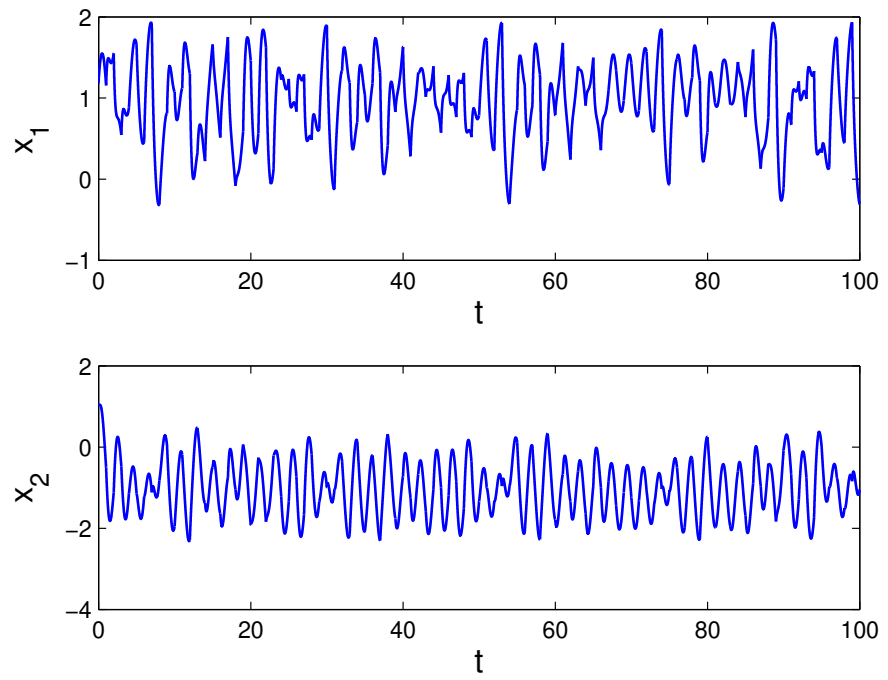

FIGURE 1. The time series of the $x_{1}$ and $x_{2}$ coordinates of system (3.3) with the initial conditions $x_{1}(0)=1,18, x_{2}(0)=1,01$. The figure manifests the irregular behavior of the system.

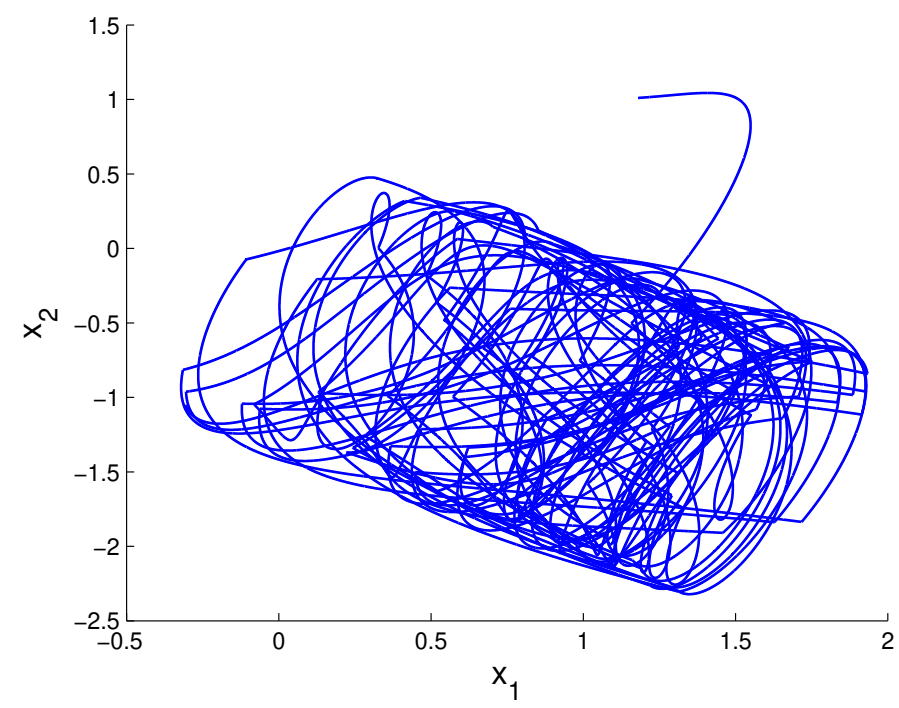

FIgURE 2. The irregular trajectory of system (3.3). The initial data $x_{1}(0)=1,18, x_{2}(0)=1,01$ are utilized in the simulation. 
The next example is devoted to a system with hyperbolic linear part such that the matrix of coefficients admit both positive and negative eigenvalues.

Example 2. Let us take into account the system

$$
\begin{aligned}
& y_{1}^{\prime}=4 y_{1}-0.21 y_{2}^{3}-2 \varphi_{2}^{3}(t) \\
& y_{2}^{\prime}=7 y_{1}-6 y_{2}-0.14 y_{1}^{2}+3 \varphi_{1}^{3}(t),
\end{aligned}
$$

where $\left(\varphi_{1}(t), \varphi_{2}(t)\right)$ is the unpredictable solution of system (3.3). One can confirm that the function $\left(-2 \varphi_{2}^{3}(t), 3 \varphi_{1}^{3}(t)\right)$ used as perturbation in system (3.4) is unpredictable in accordance with Lemma 3 , and therefore, the system possesses a unique unpredictable solution by Theorem 1. The simulation results for system (3.4) corresponding to the initial conditions $y_{1}(0)=0$ and $y_{2}(0)=0$ are shown in Figures 3 and 4. Both of the figures reveal the irregular behavior system (3.4).
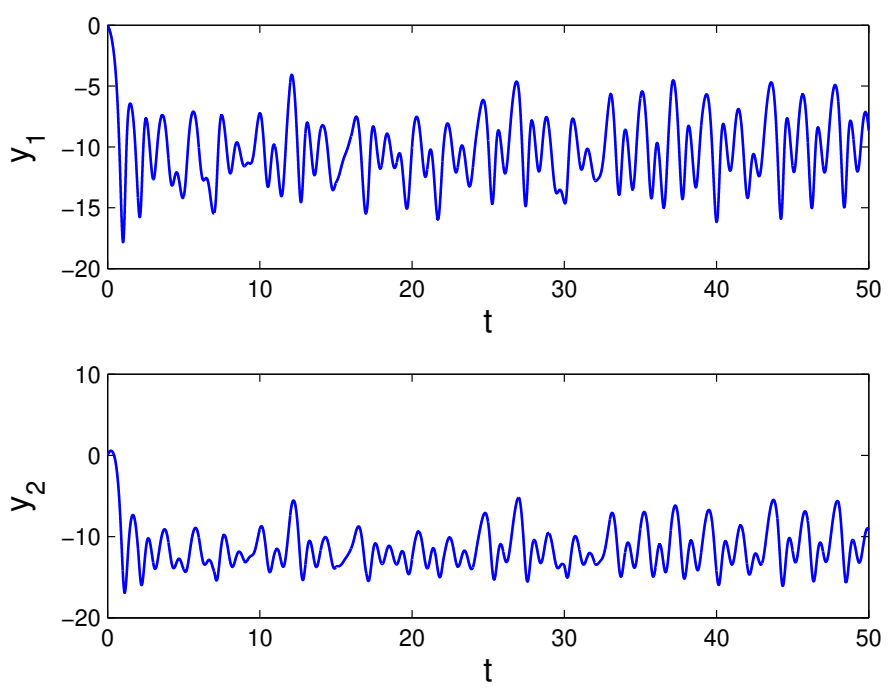

FIGURE 3. The time series for the $y_{1}$ and $y_{2}$ coordinates of system (3.4) with the initial conditions $y_{1}(0)=0, y_{2}(0)=0$. The irregular behavior of the solution reveals the presence of an unpredictable solution in the dynamics of (3.4). 


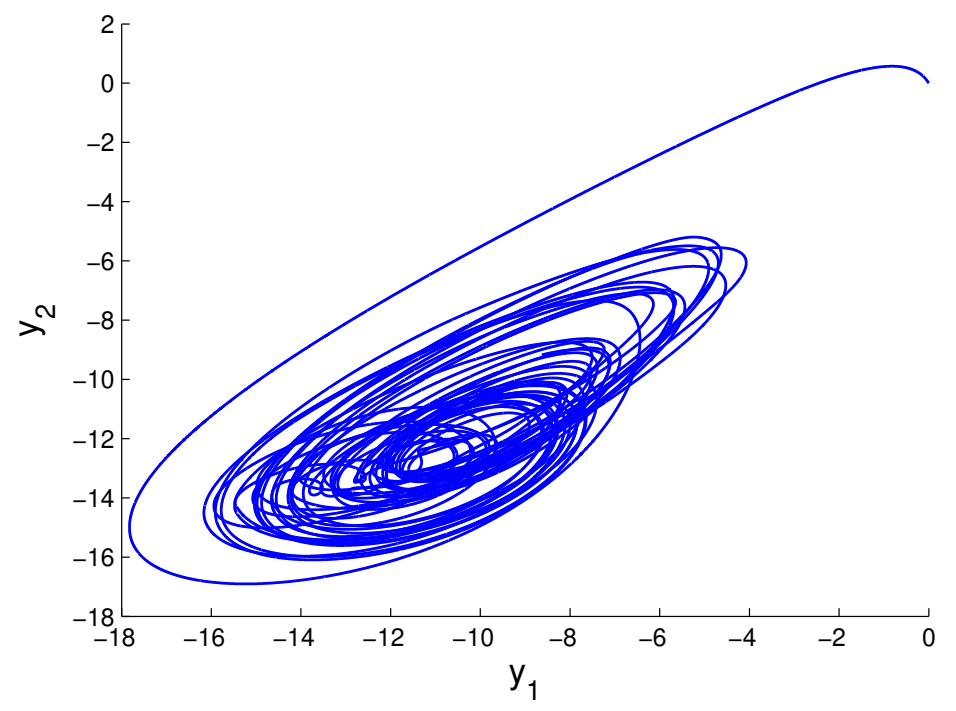

FIGURE 4. The trajectory of the solution of system (3.4). One can observe the irregular behavior of the system in the figure.

\section{ACKNOWLEDGEMENTS}

M. Akhmet and M.O. Fen have been supported by a grant (118F161) from TÜBITAK, the Scientific and Technological Research Council of Turkey.

M. Tleubergenova and A. Zhamanshin have been supported in parts by the MES RK grant No. AP05132573 "Cellular neural networks with continuous/discrete time and singular perturbations". (2018-2020) of the Committee of Science, Ministry of Education and Science of the Republic of Kazakhstan.

\section{REFERENCES}

[1] M. Akhmet and M. Fen, "Unpredictable points and chaos," Communications in Nonlinear Science and Numerical Simulation, vol. 40, pp. 1-5, 2016, doi: 10.1016/j.cnsns.2016.04.007.

[2] M. Akhmet and M. Fen, "Existence of unpredictable solutions and chaos," Turkish journal of mathematics, vol. 41, no. 2, pp. 254-256, 2017, doi: 10.3906/mat-1603-51.

[3] M. Akhmet and M. Fen, "Poincar'e chaos and unpredictable functions," Communications in Nonlinear Science and Numerical Simulation, vol. 48, pp. 85-94, 2017, doi: 10.1016/j.cnsns.2016.12.015.

[4] M. Akhmet and M. Fen, "Non-autonomous equations with unpredictable solutions," Cотmunications in Nonlinear Science and Numerical Simulation, vol. 59, pp. 657-670, 2018, doi: 10.1016/j.cnsns.2017.12.011.

[5] R. Devaney, An Introduction to Chaotic Dynamical Systems. USA: Addison-Wesley Publishing Company, 1987. doi: 10.1007/978-1-4614-6946-9.

[6] J. Hale, Ordinary Differential Equations. Malabar, FL, USA: Krieger Publishing Company, 1990.

[7] P. Hartman, Ordinary Differential Equations. New York: John Wiley, 1964. 
[8] T. Li and J. Yorke, "Period three implies chaos," Journal of Sound and Vibration, vol. 82, no. 10, pp. 985-992, 1975, doi: 10.2307/2318254.

[9] S. Wiggins, Global Bifurcation and Chaos: Analytical Methods. New York, Berlin: Springer, 1988. doi: 10.1007/978-1-4612-1042-9.

Authors' addresses

M. Akhmet

Middle East Technical University, Department of Mathematics, Ankara, Turkey

E-mail address: maratemetu.edu.tr

M.O. Fen

TED University, Department of Mathematics, Ankara, Turkey

E-mail address: monur.fenegmail.com

M. Tleubergenova

Aktobe Regional State University, Department of Mathematics, Aktobe, Kazakhstan

E-mail address: madina1970@mail.ru

\section{A. Zhamanshin}

Aktobe Regional State University, Department of Mathematics, Aktobe, Kazakhstan

E-mail address: akylbek78@mail.ru 